\title{
Numerical Investigation of a Class of Nonlinear Schrödinger Equations
}

\author{
Christopher Ventura \\ Pacific University \\ 2043 College Way \\ Forest Grove, Oregon 97116
}

Received:April 7, 2004

Accepted: July 16, 2004

\begin{abstract}
This paper numerically investigates the space-localized spherically symmetric, stationary, and singularity-free solutions of the Nonlinear Schrödinger equation when the nonlinearity is a step function. Previously no-node solutions have been obtained analytically. Here, it is shown that localized stationary solutions with one node and two nodes also exist.
\end{abstract}

\section{INTRODUCTION}

The Schrödinger equation is

$$
i \hbar \frac{\partial \psi}{\partial t}=-\frac{\hbar^{2}}{2 m} \nabla^{2} \psi+U(x) \psi
$$

where $\hbar$ is Planck's constant, $m$ is the mass of the particle, $\mathrm{U}(\mathrm{x})$ is the potential in which the particle moves, and $\psi=\psi(x, t)$ is the wave-function for that particle [1].

This is the fundamental equation of nonrelativistic quantum mechanics. It describes the motion of electrons and other elementary particles. It is a linear partial differential equation.

In contemporary physics nonlinear versions of Schrödinger equation are studied. Their form is

$$
i \frac{\partial \psi}{\partial t}=-\mu \nabla^{2} \psi+\psi G\left(\psi^{*} \psi\right)
$$

where $\mu>0$ is a real constant and $G$ is a real function of $|\psi|^{2}=\psi^{*} \psi$.

The space-localized solutions of the nonlinear Schrödinger equations are interpreted as representations of elementary particles. Only three nonlinear Schrödinger equations are known to be solvable analytically: the one with a logarithmic nonlinearity, the one with $G\left(\psi^{*} \psi\right)=k \psi^{*} \psi$ ( $k$ is a constant), and $G$ is a step function [14].
In the case when $G$ is a step function, only no-node space-localized solutions were obtained analytically. Using numerical calculations I have found one-node and twonode space-localized solutions. This is evidence that space-localized solutions of any number of nodes exist when the nonlinearity is a step function.

The goal of this paper is to numerically investigate the space localized, spherically symmetric, stationary, and singularity-free solutions of the NLS (Nonlinear Schrödinger Equation) when $\mathrm{G}$ is a step function.

\section{THE EQUATION FOR SPHERICALLY SYMMETRIC STATIONARY SOLUTIONS}

We start with equation (2). Then we use that fact that stationary solutions are of the form

$$
\psi=\varphi e^{-i \omega t}
$$

where $\varphi=\varphi(x)$ is a function of the coordinates $x=\left(x_{1}, x_{2}, x_{3}\right)$ only. By substituting equation (3) into equation (2) the NLS becomes

$$
\omega \varphi=-\mu \nabla^{2} \varphi+\varphi G\left(\varphi^{*} \varphi\right)
$$

Now we can rewrite equation (4) as the following

$$
\mu \nabla^{2} \varphi=(G-\omega) \varphi
$$




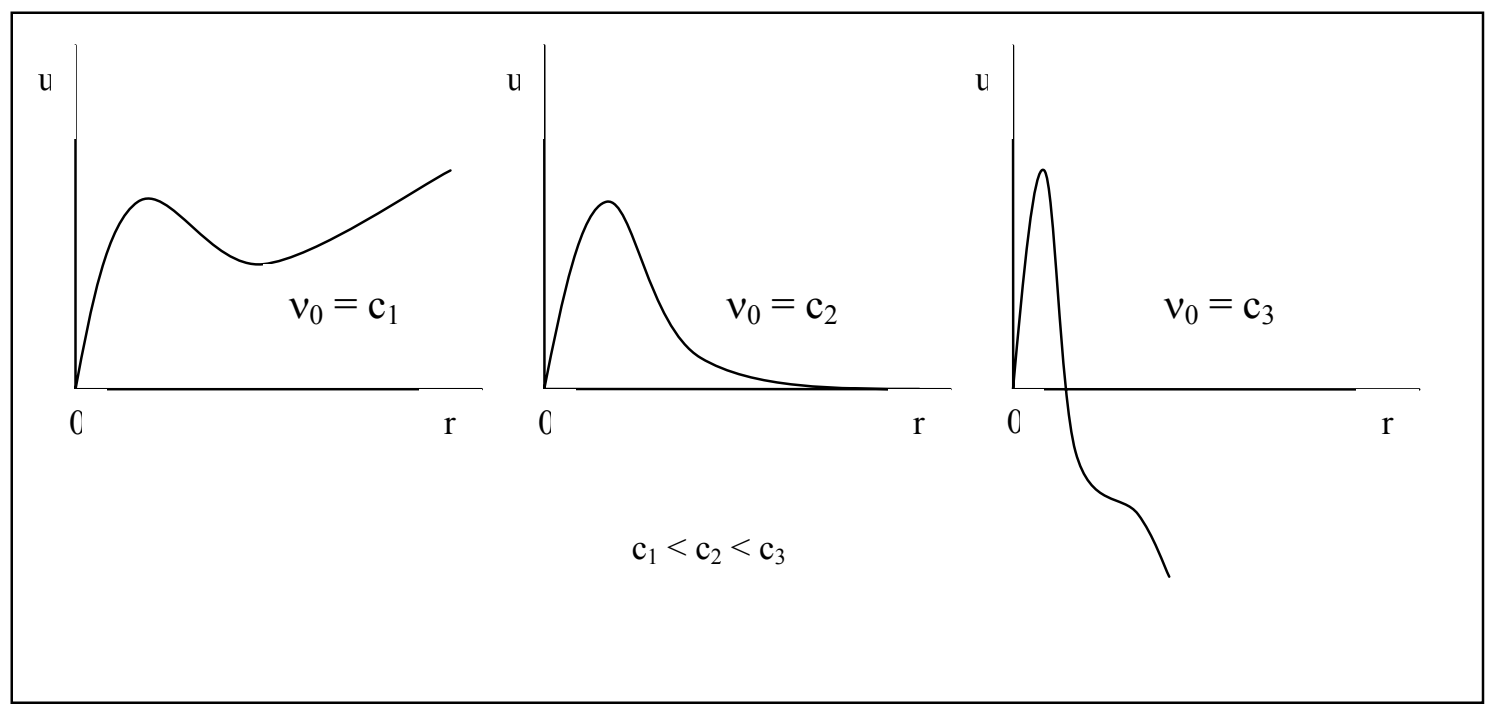

Figure 1. Three types of solution to the non-linear Schrodinger equation (NLS) for three values of the shooting parameter, $v_{0}$.

Since we are looking for spherically symmetric solutions the following must be true for $\varphi$

$$
\nabla^{2} \varphi=\frac{1}{r} \frac{d^{2}(r \varphi)}{d r^{2}}
$$

where $\varphi=\varphi(r)$ is expressed as a function of $r$.

Using this we find that our NLS becomes

$$
\frac{\mu}{r} \frac{d^{2}(r \varphi)}{d r^{2}}=(G-\omega) \varphi
$$

which is an ordinary differential equation (ODE). For our convenience we will define a new variable,

$$
u=r \varphi
$$

Now our ODE can be written as

$$
\mu \frac{d^{2} u}{d r^{2}}=(G-\omega) u
$$

Next, we define $G=G\left(\frac{|u|}{r}\right)$ to be the step function:

$$
G= \begin{cases}\omega_{0} & \frac{|u|}{r}<1 \\ 0 & \frac{|u|}{r} \geq 1\end{cases}
$$

The shooting method and Runge-Kutta method can now be used to find solutions to the following set of equations:

$$
\mid \begin{gathered}
\frac{d u}{d r}=v \\
\frac{d v}{d r}=(G-\omega) u
\end{gathered}
$$

with initial conditions

$$
u_{0}=0
$$

and $v_{O}=$ shooting parameter.

\section{THE SHOOTING METHOD}

Before the Runge-Kutta method can be applied to the NLS equation, equation (9) must be reduced to a system of two first order ordinary differential equations

$$
\begin{gathered}
\frac{d u}{d r}=v \\
\frac{d v}{d r}=\left(\frac{G}{\omega_{0}}-\omega\right) u
\end{gathered}
$$

Since the NLS equation is of order 2 , two initial conditions must be specified in order to determine a particular solution to the equations. These initial conditions are $\mathrm{u}_{0}$ and $v_{0}$. Because we are looking for singularityfree solutions, $|u| \rightarrow 0$ as $r \rightarrow 0$ (because 
$\varphi=\frac{u}{r}$ must remain finite). From this we get that $\mathrm{u}_{0}=0$. Now we have to determine the "shooting parameter" $v_{0}$. Since we are looking for space-localized solutions we know that $|u| \rightarrow 0$ as $r \rightarrow \infty$. Thus, we can just vary the parameter $v_{0}$ until a space-localized solution is found. $v_{0}$ describes the initial slope of the particular solution, and thus by varying this parameter we may "shoot" for the solution we desire (a space-localized solution). Figure 1 shows an example of three solutions obtained with three different values of the parameter $v_{0}$.

Now the Runge-Kutta method may be used to find solutions to the NLS equation.

\section{RESULTS}

First we have to verify that the shooting method works. To do this we compare the analytical result for the no-node solution with the results of the numerical method for the no-node solution. The analytical solution is

$$
\begin{aligned}
& \varphi=\frac{r_{1}}{\sqrt{\omega} r} \sin (\sqrt{\omega} r) \quad \text { for } \quad 0 \leq r \leq r_{1} \\
& \varphi=\frac{r_{1}}{r} e^{\sqrt{1-\omega}\left(r_{1}-r\right)} \quad \text { for } \quad r_{1} \leq r \leq \infty
\end{aligned}
$$

where $r_{1}$ is the value of $r$ at which the step occurs. See Georgieva and Bodurov [3] for more details on the analytical solution. Figure 2 illustrates a comparison between the numerical and analytical solutions.

\section{a. One-Node Solution:}

We have varied the parameter $\omega$, so as to include values throughout its entire domain. The shooting method was then used to determine the value $v_{0}$ for which the onenode solution exists. Figures 3,4 , and 5 represent some of these solutions for $\omega=0.1$, 0.5 , and 0.9 , respectively.

b. Two-Node Solutions:

Once again we have varied the parameter $\omega$, so as to include values throughout its entire domain. The shooting method was then used to determine the value $v_{0}$ for which the two-node solution exists. Figures 6, 7, and 8 represent some of these solutions for $\omega=0.1,0.5$, and 0.9 , respectively.

\section{V. $\quad \mathrm{L}_{2}$-NORM}

Now that we have demonstrated the existence of stationary solutions with multiple nodes, we can calculate the $L_{2}$-norm $\mathrm{N}$ of $\varphi=\frac{u}{r}$ for the one-node and two-node solutions by numerical integration. The integration is performed by applying the trapezoidal rule to $u^{2}$ at different values of $\omega$.

By the definition of the $L_{2}$-norm we have

$$
N=\int_{R^{3}} \varphi^{2} d x d y d z
$$

where $\mathfrak{R}^{3}$ stands for all space and $d x d y d z$ stands for the volume element. In our case, we have

$$
N=\int_{R^{3}} \varphi^{2} d x d y d z=\int_{0}^{\infty}\left(\frac{u}{r}\right)^{2} 4 \pi r^{2} d r=4 \pi \int_{0}^{r} u^{2} d r
$$

since $\varphi=\frac{u}{r}$ and $d x d y d z=4 \pi r^{2} d r$ because of spherical symmetry.

\section{a. No-Node}

Once again we will verify that the numerical procedure works, through comparison with the analytical solution. Figures 9 and 10 show that the $L_{z}$-norm has a single minimum at $\omega \approx 0.931$, which agrees with the results of the analytical solution.

b. One-Node

Now that we have validated the numerical procedure, we shall apply it to the one-node solutions. The results are shown in Figures 11 and 12. 

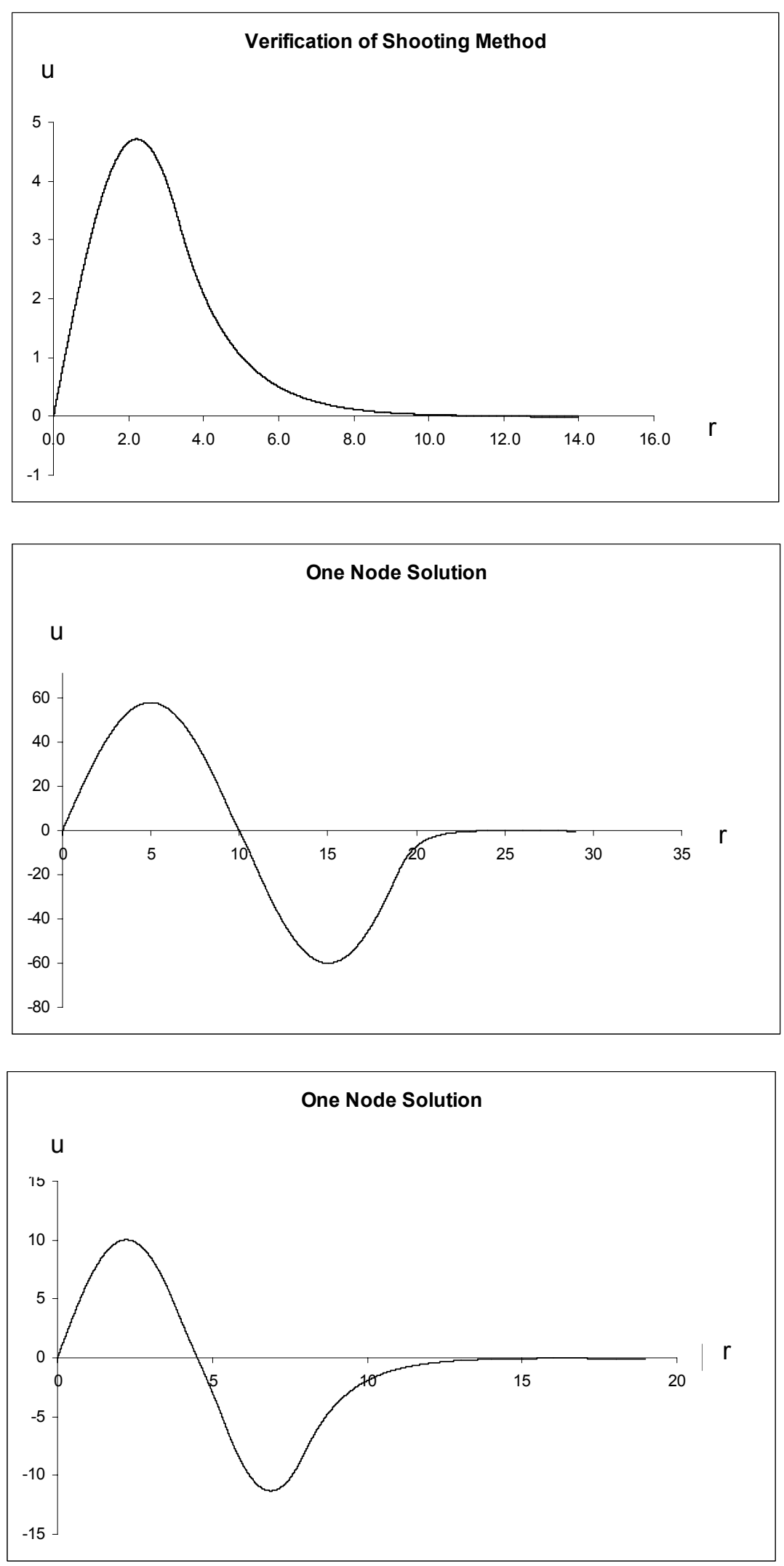

Figure 2. (Top left) Verification of the Shooting Method: A comparison between the analytical solution (solid line) and the numerical solution (dotted line) for $\omega=0.5$ and $v_{0}=3.332074$. The two solutions overlay each other quite closely.

Figure 3. (Middle left) An example of a OneNode solution, with $\omega$ $=0.1$ and $v_{0}=$ 18.331585

Figure 4. (Bottom left) An example of a OneNode solution, with $\omega$ $=0.5$ and $v_{0}=$ 7.088522 . 
Figure 5. (Top right) An example of a OneNode solution, with $\omega$ $=0.9$ and $v_{0}=$ 4.277392 .

Figure 6. (Middle right) A Two-Node solution, with $\omega=0.1$ and $v_{0}=27.653563$.

Figure 7. (Bottom right) A Two-Node solution, with $\omega=0.5$ and $v_{0}=10.778026$.
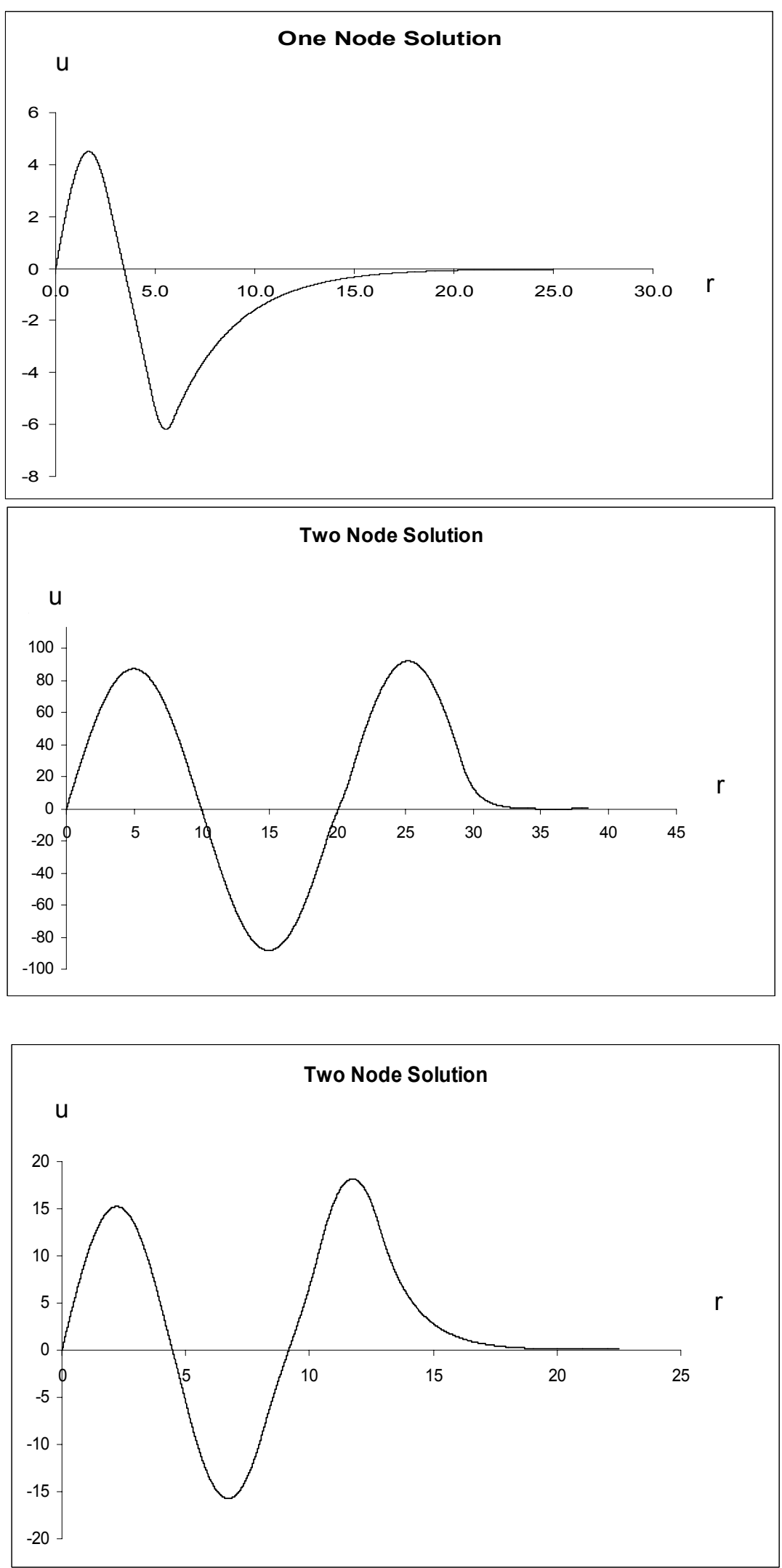

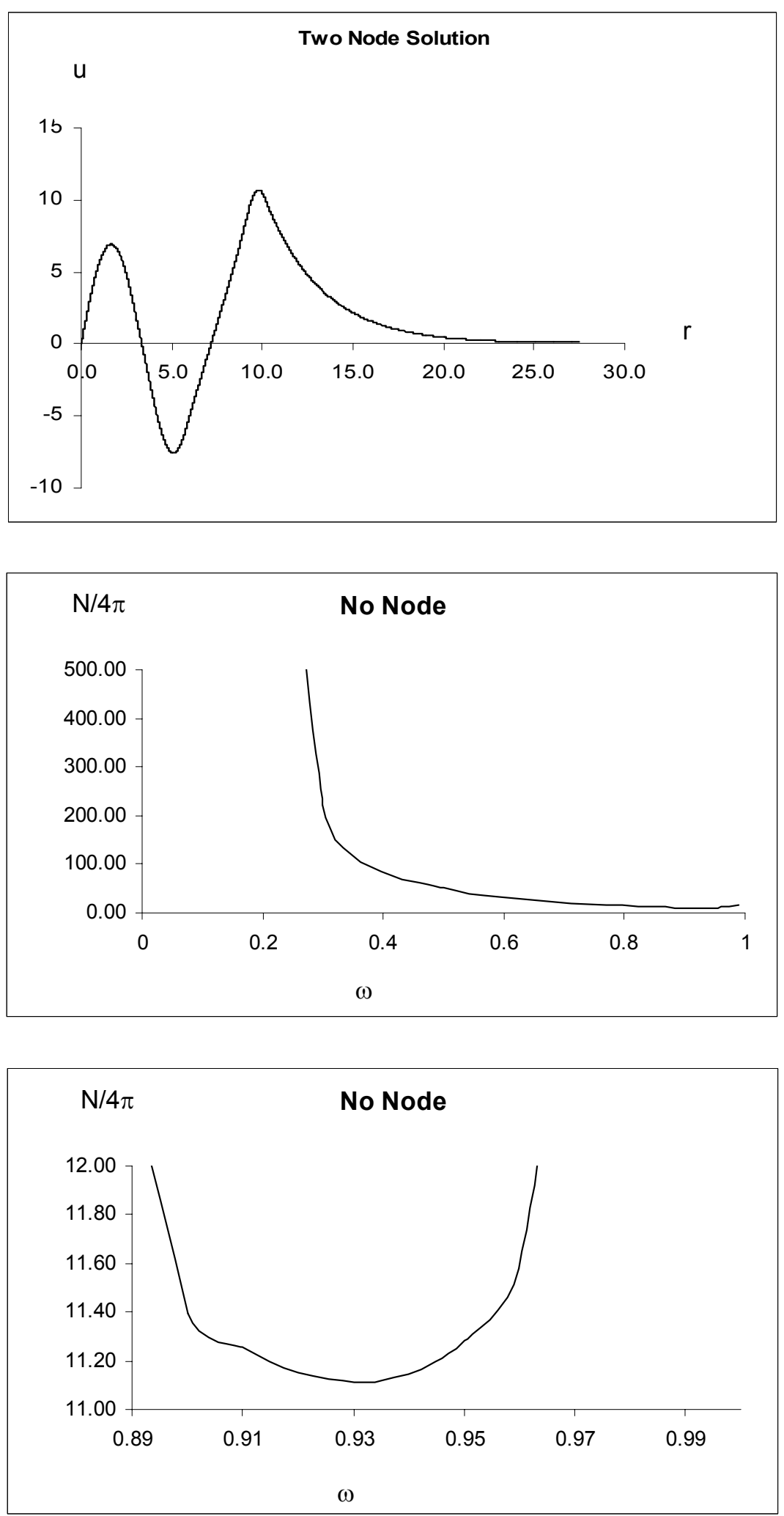

Figure 8. (Top left) A Two-Node solution, with $\omega=0.9$ and $v_{0}=$ 6.562161 .
Figure 9 (Middle left) and Figure 10 (Bottom left) show that the $\mathrm{L}_{z^{-}}$ norm has a single minimum in the NoNode case at $\omega \approx$ 0.931, which agrees with the results of the analytical solution. 
Figures 11 and 12 show that the $L_{2}$-norm has a single minimum.
c. Two-Node

Now we shall apply the procedure to the two-node solutions. The results are shown in Figures 13 and 14.
Figure 11 (Top right) and Figure 12 (Middle right) show that the $L_{2-}$ norm also has a single minimum in the case of the One-Node solutions.
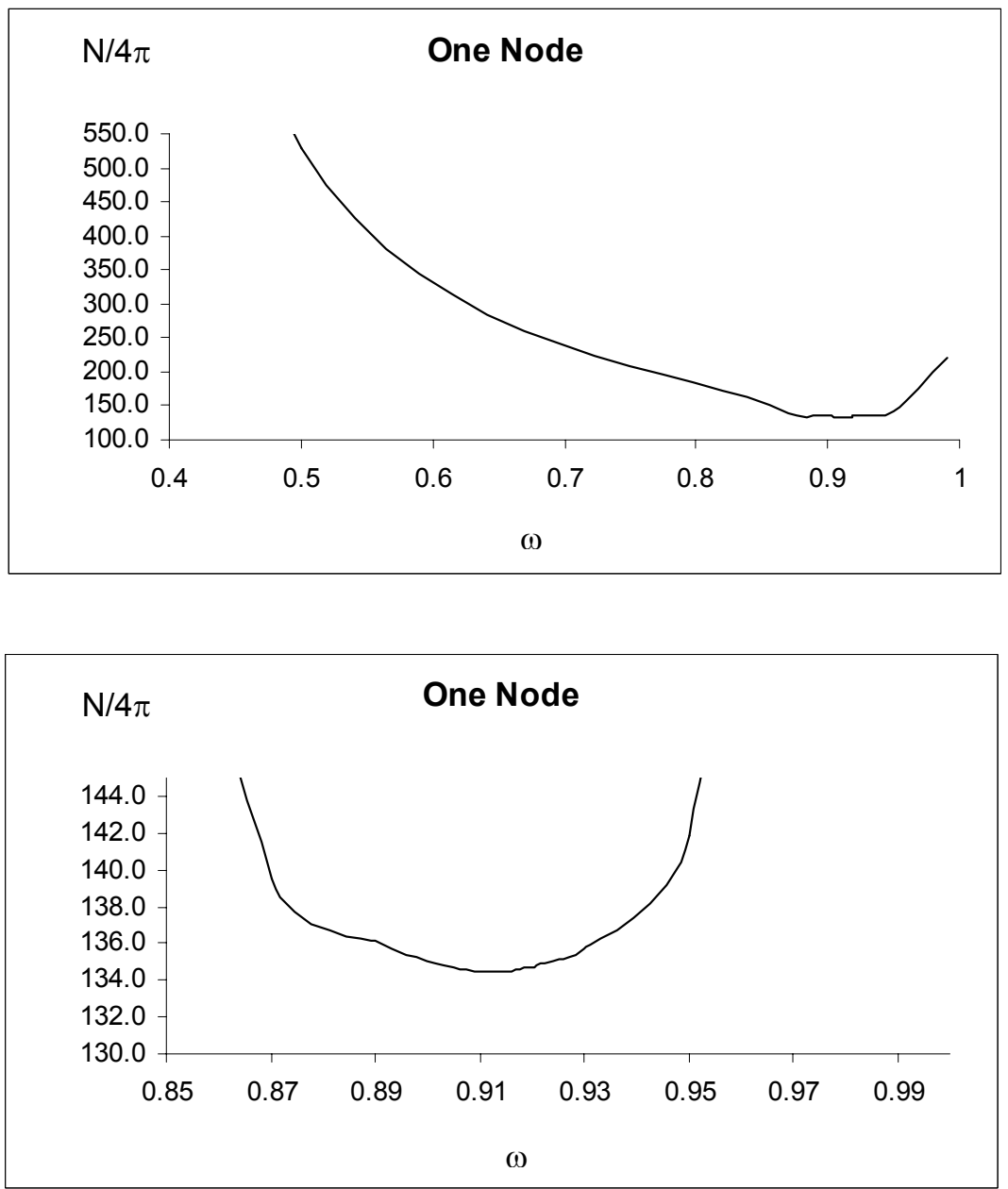

Figure 13 (Bottom right) and Figure 14 (Top next page) show that the $\mathrm{L}_{2}-$ norm also has a single minimum in the case of the Two-Node solutions.

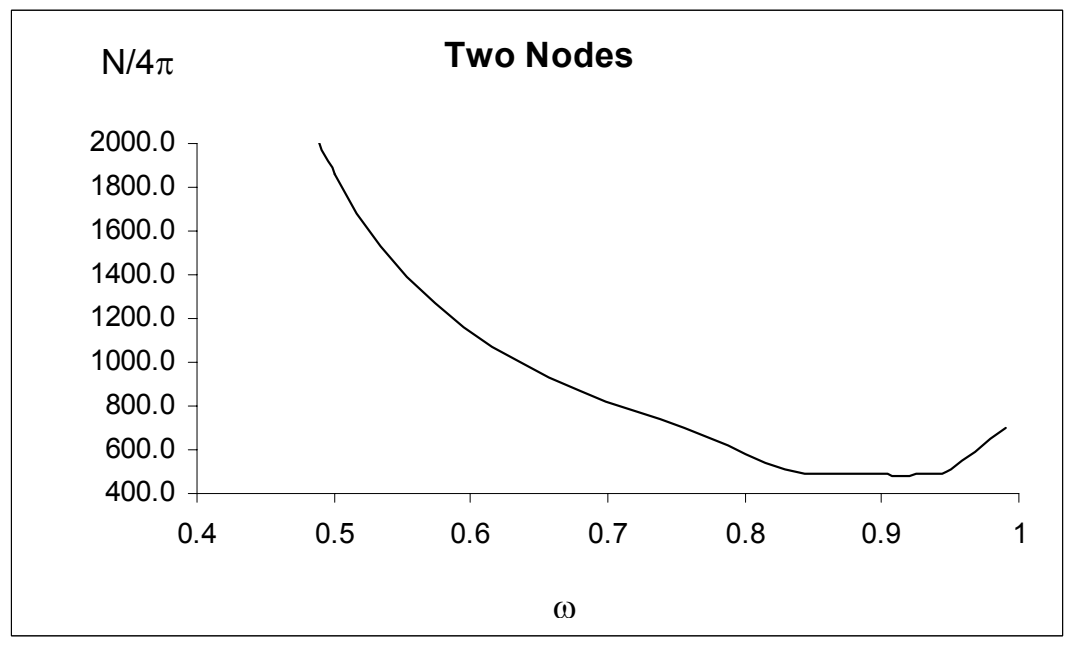




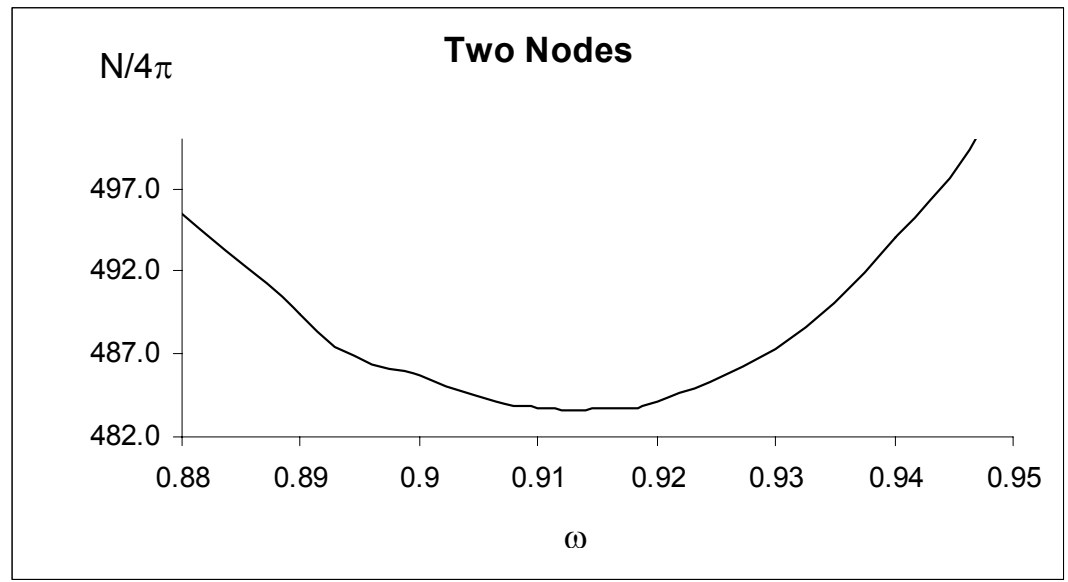

Figure 14 (at left) shows that the $\mathrm{L}_{2^{-}}$ norm also has a single minimum in the case of the Two-Node solutions.

\section{CONCLUSION}

The above results are presented as evidence that space-localized solutions of one node and two nodes exist, when the non-linearity in the NLS is a step function. Based on the results of this paper, we can conjecture that solutions with any number of nodes exist. We have also demonstrated that the nonode, one-node, and two-node solutions all have a single minimum for their $L_{2}$-norm.

\section{ACKNOWLEDGEMENT}

I am very grateful to Professor Bogdana Georgieva who suggested this project and guided me to its successful end.

\section{REFERENCES}

1. Bialynicki-Birula, I., Mycielski, J.. "Nonlinear Wave Mechanics", Annals of Physics, 1000 (1976), pp. 62-93.

2. A. Filippov, Differential Equations With Discontinuous Righthand Sides (Kluwer Academic, New York, 1988).

3. B. Georgieva, T. Bodurov. "Nonlinear Schrödinger equation whose nonlinearity is a step function", International Journal of Differential Equations and Applications Vol. 7 (2003), No.4, pp. 443-452.

4. H. Berestycki, P. Lions. "Nonlinear Scalar Equations", Archive for Rational Mechanics and Analysis, 82 (1983), No. 4, pp. 313-375.
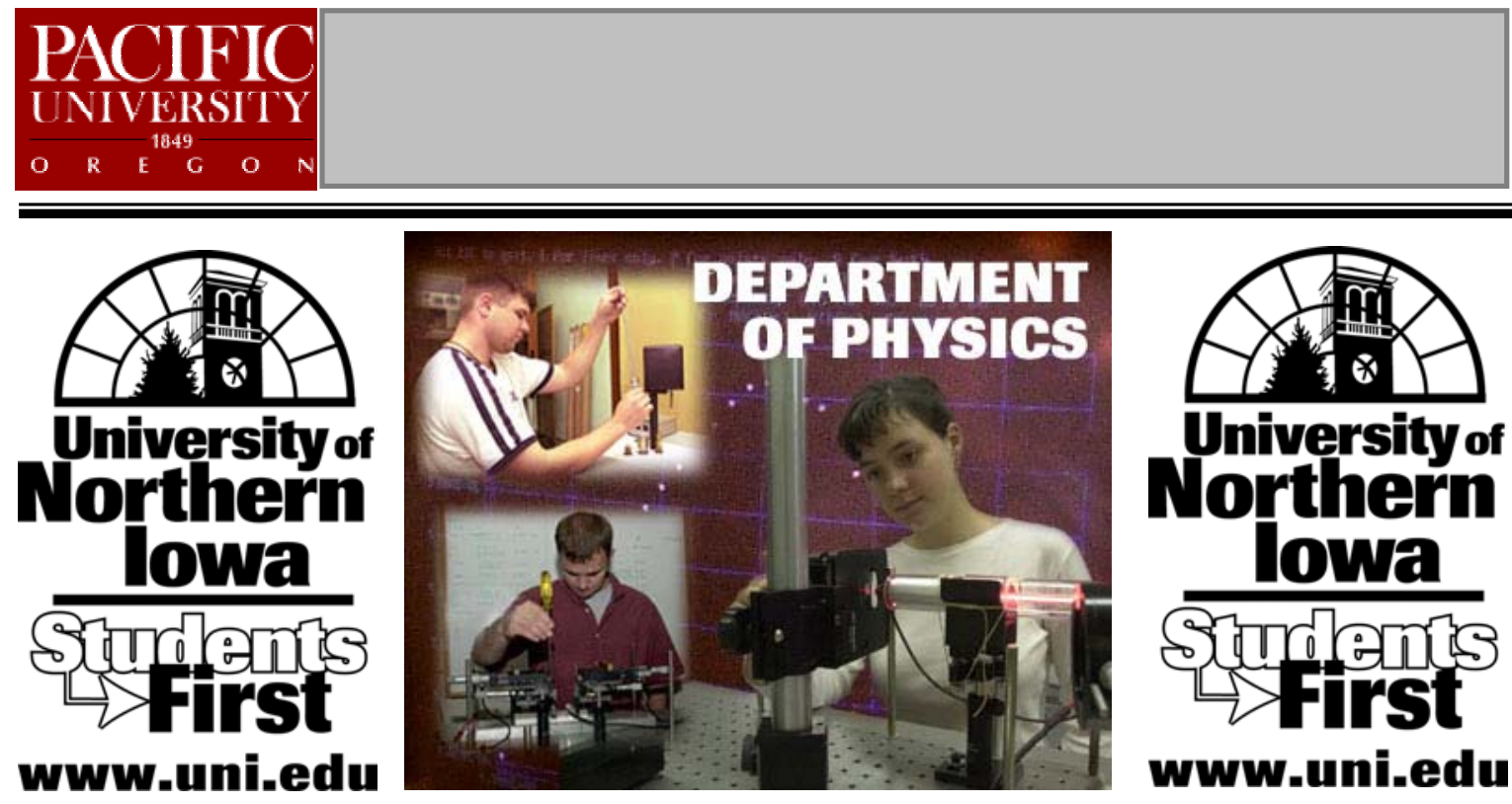

www.uni.edu 